Disponível em

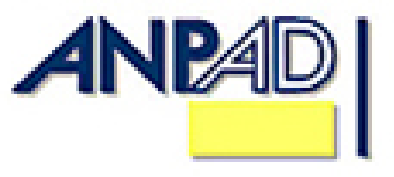

http://www.anpad.org.br/rac

RAC, Rio de Janeiro, v. 16, n. 2, art. 4, pp. 237-252, Mar./Abr. 2012

(oc) EY-No

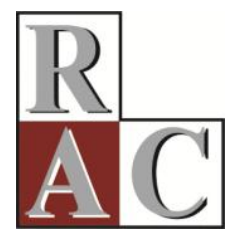

\title{
Eficiência Relativa da Política Nacional de Procedimentos Cirúrgicos Eletivos de Média Complexidade
}

\author{
Relative Efficiency of National Policy for Elective, Medium-Complexity Surgical \\ Procedures
}

\begin{abstract}
Marcel de Moraes Pedroso *
E-mail: marcelpedroso@unb.br Universidade de Brasília - PPGA/UnB Brasília, DF, Brasil.

Paulo Carlos Du Pin Calmon

E-mail: paulo.calmon@gmail.com Universidade de Brasília - PPGA/UnB

Brasília, DF, Brasil.

Ludmila Ferreira Bandeira E-mail: ludmilafbandeira@gmail.com Universidade de Brasília - PPGA/UnB Brasília, DF, Brasil.

Rogéria Aparecida Valter de Lucena E-mail: rogeria.lucena@ saude.gov.br Coordenação Geral de Média e Alta Complexidade do Ministério da Saúde - CGMAC/DAE/SAS/MS Brasília, DF, Brasil.
\end{abstract}

\footnotetext{
* Endereço: Marcel de Moraes Pedroso

BCE - Biblioteca Central, Campus Universitário Darcy Ribeiro Gleba "A", Sala 312, Brasília/DF, 70910-900.
}

Copyright (C) 2012 RAC. Todos os direitos, até mesmo de tradução, são reservados. É permitido citar parte de artigos sem autorização prévia, desde que seja identificada a fonte. 


\title{
Resumo
}

O trabalho avalia a eficiência relativa das Unidades Federativas na gestão da Política Nacional de Procedimentos Cirúrgicos Eletivos de Média Complexidade por meio da utilização de Análise Envoltória de Dados (Data Envelopment Analysis [DEA]), para o ano de 2006. A modelagem DEA foi desenvolvida para determinar a eficiência de unidades tomadoras de decisão (Decision Making Units [DMUs]), em situações nas quais não seja relevante ou não se deseje considerar somente o aspecto financeiro. Foram selecionados dados e indicadores relativos às despesas totais de política, leitos cirúrgicos, médicos cirurgiões (inputs), população (uncontrolled input) e produção hospitalar de cirurgias eletivas (como output). $\mathrm{O}$ artigo apresenta os valores observados e esperados para as variáveis propostas, e sugere o nível de esforço necessário para que a UF se desloque para a fronteira de eficiência relativa. A utilização da metodologia ilustra as potencialidades da Análise Envoltória de Dados como técnica alternativa aos modelos econométricos para avaliação de políticas públicas, contratualização de metas e priorização de ações corretivas, na medida em que identifica, por variável de interesse, as folgas ou níveis de esforços necessários para o alcance de patamares mais eficientes de gestão.

Palavras-chave: avaliação de políticas públicas; governança; eficiência relativa; análise envoltória de dados.

\begin{abstract}
This paper evaluates the relative efficiency of Brazilian states (and the federal district of Brasilia) in the management of a National Policy for Elective, Medium-Complexity Surgical Procedures by using a Data Envelopment Analysis - DEA, for the year 2006. The DEA model was developed to determine the efficiency of Decision Making Units - DMUs, when it is neither relevant nor desirable to consider only financial aspects. Data and indicators used were related to the total costs of the National Policy: surgical beds, surgeons (inputs), population (uncontrolled input) and elective surgery hospital production (as an output). This article presents values that were both observed and expected for the proposed variables and suggests the level of effort required so that each state may move towards relative efficiency. The use of this methodology illustrates the potential DEA has as an alternative technique to econometric models for evaluating public policies, the setting of goals, and the prioritization of corrective actions when variables show that there are gaps or further effort required to attain more efficient management standards.
\end{abstract}

Key words: evaluation of public policies; governance; relative efficiency; data envelopment analysis. 


\section{Introdução}

Avaliar a gestão de programas governamentais é uma tarefa importante, mas de difícil implementação. A ausência de práticas avaliativas dificulta o aperfeiçoamento dos programas e o controle político e social, o que é fundamental nas democracias contemporâneas. Por outro lado, a atribuição de valor ou mérito à gestão de um determinado programa deve ser embasada em uma estratégia metodológica sólida e na demanda de tempo e recursos, elementos que nem sempre estão disponíveis no setor público. Surge, portanto, o desafio de desenvolver estratégias consistentes para avaliação, as quais gerem análises que contribuam para o aperfeiçoamento da gestão de programas.

O propósito desse artigo ${ }^{(1)}$ é apresentar os resultados de uma avaliação que faz uso de uma técnica simples, a qual demanda uma quantidade relativamente pequena de dados. Esta foi capaz de gerar informações úteis para a análise da gestão da Política Nacional de Procedimentos Cirúrgicos Eletivos de Média Complexidade em diferentes unidades da federação. Cabe notar que, embora denominada política, as iniciativas do Ministério da Saúde que são objeto de análise neste artigo têm características organizacionais e operacionais típicas de um programa governamental.

Políticas públicas são decisões. Uma política pública se constitui naquilo que o governo decide fazer ou não. É o que o Estado faz em resposta a desafios políticos. Um programa governamental consiste em todas as atividades concebidas para implementar uma política pública. Frequentemente essas atividades requerem a criação de organizações públicas, agências governamentais ou ministérios que, por sua vez, precisam estabelecer novas políticas que orientem a atuação dos agentes públicos e privados sobre como implementar as políticas públicas em si (Shafritz, Russell, \& Borick, 2007, p. 42).

Em outras palavras, as políticas públicas representam orientações mais gerais, que são implementadas mediante um conjunto de ações específicas, por meio de programas governamentais. Assim, a decisão de considerar a saúde como um direito de todos e um dever do Estado, conforme estabelecido no Artigo XXX da Constituição Federal, orienta a política de saúde pública no Brasil. Ela é concretizada mediante um amplo conjunto de programas governamentais que contemplam a atenção básica (Ex. Estratégia Saúde da Família), a assistência emergencial (Ex. Serviço Móvel de Urgência e Emergência [SAMU], e Unidades de Pronto Atendimento [UPAs]) e as intervenções da atenção especializada (Ex. Política Nacional de Procedimentos Cirúrgicos Eletivos de Média Complexidade).

A Política Nacional de Procedimentos Cirúrgicos de Média Complexidade é parte do esforço de estabelecimento do Sistema Único de Saúde (SUS), que se constitui como um dos maiores sistemas públicos de saúde no mundo. O SUS está sendo estabelecido com base em dois compromissos fundamentais: (a) a universalização dos serviços de saúde, garantindo acesso a toda a população do país; e (b) a descentralização desses serviços, atribuindo aos gestores estaduais e municipais importantes responsabilidades e autonomia no planejamento e na execução das ações voltadas para a saúde pública.

O SUS possui um sistema de governança complexo, fundamentado na atuação de diversos órgãos colegiados, em especial as comissões chamadas bipartites (estados e municípios) e tripartites (incluem também o governo federal), que gerenciam as ações das redes de atendimento e decidem sobre a alocação de recursos no Sistema ${ }^{(2)}$.

Nesse contexto, a Política Nacional de Procedimentos Cirúrgicos Eletivos de Média Complexidade é um programa gerenciado pelo Ministério da Saúde, mas implementado localmente, com intuito de proporcionar serviços que se complementem ao atendimento proporcionado pelas redes de atenção básica, pilar principal do SUS.

Historicamente, a avaliação formal de políticas públicas teve como marco o período que vai da Grande Depressão nos Estados Unidos até o período que sucedeu a Segunda Guerra Mundial, pela necessidade de avaliar políticas implementadas para equacionar os problemas sociais emergentes. A 
expansão do campo da avaliação deu origem a diferentes vertentes e tendências que, resumidamente, podem ser divididas em quatro gerações: a primeira (1910-1930), que tem como ênfase a construção e a aplicação de instrumentos de medidas para avaliar os beneficiários de uma intervenção; a segunda (1930-1967), centrada na descrição da intervenção, marca o surgimento da avaliação de programas; a terceira (1967-1980), apoiada no julgamento de mérito e do valor de uma intervenção para ajudar na tomada de decisões; e a quarta (1980 - atual), refere-se à negociação entre os atores interessados e envolvidos na avaliação (Almeida, 2006; Faria, 2005; Figueiró, Frias, \& Navarro, 2010; Guba \& Lincoln, 1989).

Os programas implementados no âmbito do SUS têm sido objeto de diversas avaliações, que fazem uso de diferentes abordagens como, por exemplo, técnicas de análise de custo-benefício (Machado \& Simões, 2008) ou a tentativa de caracterizar a Economia Política da Saúde (Reis, Ribeiro, \& Piola, 2001). A abordagem aqui proposta é distinta, pois objetiva analisar a eficiência relativa da gestão descentralizada da Política nas diferentes Unidades da Federação mediante a utilização da técnica de Análise Envoltória de Dados (Data Envelopment Analysis [DEA]). Trata-se de técnica de implementação relativamente simples e capaz de gerar informações úteis que permitem analisar comparativamente os resultados da gestão do programa em diferentes UFs.

De acordo com Marinho, Cardoso e Almeida (2011), a utilização da metodologia DEA para análise de sistemas de saúde no Brasil vem crescendo (Calvo, 2005; La Forgia \& Couttolenc, 2008; Marinho, 2000, 2001, 2003; Marinho \& Façanha, 2002; Proite \& Souza, 2004). No exterior, a utilização da DEA na avaliação do setor de saúde é mais antiga e mais frequente (Afonso \& St. Aubyn, 2005; Chilingerian, 1994; Hadad, Hadad, \& Simon-Tuval, in press; Joumard, Andrée, \& Nicq, 2010; Parkin \& Holligsworth, 1997; Rouse, Harrison, \& Chen, 2010; Smith \& Street, 2005).

Cabe ressaltar que a aplicação da DEA viabiliza a comparação do desempenho das Unidades da Federação, atribuindo valor ou mérito relativo e não absoluto à gestão do programa. Essa comparação gera informação de fácil utilização na medida em que favorece a melhor compreensão dos problemas e a identificação das melhores práticas.

\section{A Política Nacional de Procedimentos Cirúrgicos Eletivos de Média Complexidade}

Os Mutirões Nacionais de Cirurgias Eletivas (cirurgias de catarata, varizes, próstata, retinopatia diabética) foram implantados em 1999 pelo Ministério da Saúde em parceria com os estados e municípios. Os recursos financeiros destinados para a execução desta ação eram disponibilizados pelo Fundo de Ação Estratégica e Compensação (FAEC), por intermédio de portarias publicadas periodicamente, habilitando os estados e municípios - sob gestão plena - a executar os mutirões.

De acordo com levantamento do Ministério da Saúde, sobre prevalência e cirurgias eletivas realizadas, pode-se inferir que, antes da implantação dos mutirões, a demanda potencial estimada (necessidade) era bastante superior ao número de procedimentos cirúrgicos realizados anualmente. Com a implantação dos mutirões, em 1999, tal demanda foi gradualmente atendida e, a partir do ano 2000, tem apresentado tendência declinante (Ministério da Saúde [MS], 2008).

Em 2003, a produção nacional para cirurgia de catarata passou a ser maior que a necessidade, havendo um atendimento de toda a fila de espera do país. Observou-se, pois, que a demanda reprimida nacional existente estava atendida, mantendo-se apenas a rotina (casos novos). Porém, os mutirões não foram capazes de desempenhar papel estruturante na organização das redes de serviços. Assim, a ocorrência de novos cenários com demanda reprimida para este tipo de cirurgia não foi esgotada (MS, 2008).

No entanto, por terem sido tratados como uma demanda nacional, os mutirões levaram à redução (em alguns casos à extinção) das filas de espera. Porém, como eram restritos a um reduzido grupo de cirurgias (procedimentos), outras demandas não foram solucionadas, tendo como 
consequência o surgimento de filas em outras cirurgias eletivas relevantes em âmbito local. Dessa forma, o planejamento de uma estratégia de implantação de uma política nacional de cirurgias eletivas se fez necessário com o objetivo de reduzir a fila de espera para outros tipos de cirurgias, considerando o perfil epidemiológico e a organização geral do sistema.

Como os mutirões nacionais não atingiram um de seus principais objetivos (organização da rede), o Ministério da Saúde, por intermédio da Secretaria de Atenção à Saúde/Departamento de Atenção Especializada/Coordenação de Média Complexidade Ambulatorial (MS/SAS/DAE/CGMAC), instituiu a Política Nacional de Procedimentos Cirúrgicos Eletivos de Média Complexidade.

Em 2006, as estratégias dos mutirões nacionais foram encerradas com a redefinição da Política Nacional de Procedimentos Cirúrgicos Eletivos de Média Complexidade (Portaria n. 252, 2006) alinhada às diretrizes do Pacto pela Saúde (Portaria n. 399, 2006).

No âmbito do SUS, a implantação da Política Nacional de Procedimentos Cirúrgicos Eletivos de Média Complexidade está inserida em um processo de planejamento global orientado pelos princípios e diretrizes da Lei n. 8.080 (1990). Esse processo envolve a garantia da universalidade e a oportunidade de acesso dos cidadãos a todas as ações e serviços necessários, a integralidade da atenção e a equidade na alocação dos recursos de forma coerente e global.

A Política foi construída visando promover a estruturação da rede. É executada mediante apresentação, por parte dos entes federados, de Projeto $^{(3)}$ ao Ministério da Saúde. A Política possui os seguintes objetivos: (a) dar autonomia aos gestores municipal e estadual para definição dos procedimentos cirúrgicos eletivos de média complexidade a serem realizados com recursos extrateto ${ }^{(4)}$, responsabilizando os mesmos pelo controle e avaliação da execução destes procedimentos; (b) estruturar uma rede de serviços regionalizada e hierarquizada que permita cuidados integrais de saúde e melhoria do acesso ao atendimento especializado de cirurgias eletivas de média complexidade; (c) identificar a demanda reprimida e reduzir filas de espera por procedimentos cirúrgicos eletivos de média complexidade, possibilitando a organização local do sistema e a ampliação, se necessário, da oferta desses serviços (MS, 2009).

Os recursos financeiros previstos para a execução dos projetos são transferidos de forma regular e automática do Fundo Nacional de Saúde (FNS) aos Fundos Municipais e Estaduais de Saúde. São calculados a partir da população total de abrangência do ano anterior, com base nas estimativas de distribuição das cotas do Fundo de Participação dos Municípios (FPM) realizadas pelo Tribunal de Contas da União (TCU), com um valor per capita/ano de R\$2,25. Os recursos integram o bloco de financiamento para a gestão do Sistema Único de Saúde, no componente Incentivo à Implementação de Políticas Específicas, conforme estabelecido no Pacto pela Saúde.

Os limites financeiros para a execução dessa Política são calculados para 12 meses. Os projetos apresentados devem conter metas (com limites financeiros proporcionais ao período) para execução. Caso as metas propostas sejam cumpridas, os gestores poderão apresentar novo projeto com metas para o ano subsequente. Para garantir que o repasse seja efetuado de maneira objetiva, a Comissão Intergestores Bipartite $(\mathrm{CIB})^{(5)}$ deve discriminar como será feito o repasse financeiro ao município, a fim de que este execute o que foi proposto no Projeto encaminhado.

Os municípios e estados que apresentarem projetos para a realização de procedimentos cirúrgicos eletivos de média complexidade deverão identificar os nomes dos municípios adstritos, bem como a população de abrangência, com seus respectivos valores financeiros. É imprescindível constar esta relação em declaração da CIB (ver Tabela 2 para informações sobre número de projetos e número de municípios envolvidos por UF). 


\section{Análise Envoltória de Dados}

As ideias básicas sobre Análise Envoltória de Dados (Data Envelopment Analysis [DEA]) foram desenvolvidas pelo artigo de Farrell (1957), que almejava corrigir as limitações encontradas nos índices de produtividade até então utilizados e substituí-los pela noção de eficiência relativa, baseada na análise de atividade de Debreu (1951). O trabalho de Farrell despertou pouca atenção até a publicação do texto clássico de Charnes, Cooper e Rhodes (1981), posteriormente modificado por Banker, Charnes e Cooper (1984).

A DEA consiste em uma metodologia não paramétrica, ou seja, não exige uma forma funcional explícita relacionando as variáveis para mensuração comparativa da eficiência de Unidades Tomadoras de Decisão (Decision Making Units [DMUs]), com base nas melhores práticas. Essa técnica permite analisar a eficiência relativa de unidades produtivas com múltiplos insumos (inputs) e múltiplos produtos (outputs) por intermédio da construção de uma fronteira virtual de eficiência e da identificação de ineficiências geradas por decisões e ações subótimas (ver Figura 1).

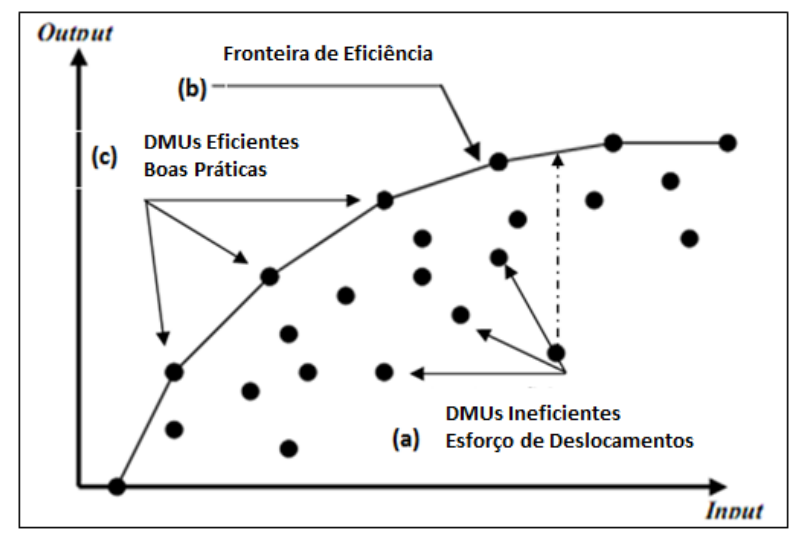

Figura 1. Esquema Gráfico Básico da DEA.

Fonte: Elaborado com adaptações do trabalho de Lins, M. P. E., Lobo, M. S. C., Silva, A. C. M., Fiszman, R., \& Ribeiro (2007). O uso da análise envoltória de dados (DEA) para avaliação de hospitais universitários brasileiros. Ciência \& Saúde Coletiva, 12(4), 985-998. p. 989.

A Figura 1 representa o esquema gráfico básico da DEA: as DMUs ineficientes (a) estão delimitadas por um conjunto de referência (fronteira do envelope ou envoltória) de unidades eficientes ou benchmarks; (b) o modelo é baseado na resolução de um problema de programação fracionária, no qual a medida de eficiência é obtida através da razão da soma ponderada dos produtos pela soma ponderada dos insumos. Esta é formada pela combinação linear que conecta os conjuntos de observações das unidades eficientes ou boas práticas, (c) gerando um conjunto convexo de possibilidades de produção.

Assim, o valor de eficiência atribuído à unidade não é definido como um padrão absoluto, mas sim relativo às outras unidades. As unidades que possuírem a melhor relação insumo ponderado/produto ponderado serão consideradas mais eficientes e estarão situadas sobre a linha da fronteira. Já as menos eficientes estarão situadas em uma região abaixo da fronteira e, portanto, precisarão realizar algum esforço para se deslocar para o limite de eficiência (Ferreira \& Pitta, 2008). Os modelos DEA fazem a agregação de inputs, transformando-os em insumos esperados, e a agregação de outputs, transformando-os em produtos esperados, resultantes de uma combinação linear dos inputs e outputs observados.

As DMUs (no nosso caso as Unidades Federativas [UF]) são comparadas por indicadores de inputs e outputs, gerando uma combinação convexa entre todas as outras unidades. Desse modo, uma DMU será classificada como relativamente eficiente se nenhuma outra, isolada ou em combinação com outras, puder suplantá-la em qualquer um de seus níveis de saída sem prejudicar qualquer outro 
nível de saída, nem despender mais em pelo menos um de seus níveis de entrada (eficiência de Pareto). Segundo Castro (2003), a utilização de DEA torna possível: (a) determinar quantitativamente a eficiência relativa de cada DMU; (b) identificar origens e quantidades de ineficiência relativa em cada uma das DMU, em qualquer uma das variáveis ou em qualquer uma de suas dimensões inputs ou outputs; (c) apoiar o planejamento de metas (nível de esforço necessário para o alcance da fronteira) para as diversas variáveis e dimensões que maximizem a eficiência de cada unidade.

A operacionalização da metodologia DEA segue três etapas principais (Lins \& Meza, 2000): (a) definição e seleção das DMUs; (b) escolha do modelo DEA apropriado (retornos constantes de escala ou retornos variáveis de escala); e (c) seleção das variáveis (inputs e outputs) que são relevantes para estabelecer a eficiência relativa entre as DMUs selecionadas. A seleção de unidades para a análise é de grande importância para os resultados, já que a metodologia DEA é sensível a valores extremos (Faria, Jannuzzi, \& Silva, 2008), o que configura, ao mesmo tempo, uma limitação (se os dados forem imprecisos) e uma potencialidade (se os dados denotam situações em que ocorrem boas práticas).

Por isso é necessário garantir que os indicadores relativos às DMUs sejam confiáveis, e que eventuais variações extremas sejam, de fato, situações concretas, não erros de medida ou de registro. Ou seja, os valores que se apresentam muito afastados da tendência central dos indicadores em questão podem não ser potenciais outliers, e sim um padrão a ser seguido pelas unidades ineficientes (benchmark) para que se tornem eficientes. Por isso, antes da aplicação da metodologia é necessário realizar uma análise exploratória de dados, com o intuito de avaliar DMUs discrepantes (Lins \& Meza, 2000).

\section{Aplicação do Modelo e Principais Resultados}

As DMUs selecionadas para o estudo foram as 23 Unidades Federativas (UF) que apresentaram projetos em 2006, entendidas como Unidades Tomadoras de Decisão (DMU) autônomas. Estas desempenham funções de gestão descentralizada semelhantes e que são mensuradas por uma metodologia homogênea e, portanto, são comparáveis.

A definição pelo modelo ${ }^{(6)}$ com retornos variáveis de escala, de acordo com o objetivo proposto e a literatura sobre o tema, deve-se ao fato de que, em geral, as relações que se estabelecem na gestão de programas e políticas públicas não pressupõem retornos constantes de escala. Tal modelo utiliza a fronteira VRS (variable returns to scale), que considera rendimentos variáveis de escala, com orientação output. Trata-se, pois, de maximizar os outputs sem alterar os inputs.

O modelo com retornos variáveis de escala permite que DMUs que operam com baixos valores de inputs tenham retornos crescentes de escala, e as que operam com altos valores tenham retornos decrescentes de escala. Fornece como resultados o ranking das eficiências por UF e o potencial de melhoria de todos os inputs e outputs para cada DMU.

A escolha das variáveis (ver Tabela 1) procurou traduzir os aspectos mais importantes sobre a gestão descentralizada da Política, monitorados pelo Ministério da Saúde, por intermédio de relatórios trimestrais e de acompanhamentos da execução/produção por tabulações do Departamento de Informática do SUS (DATASUS, Recuperado em 18 junho, 2010, de http://tabnet.datasus.gov.br/cgi/deftohtm.exe?sih/cnv/qruf.def). 
Tabela 1

\section{Descrição Sintética das Variáveis Utilizadas no Estudo}

\begin{tabular}{ccccc}
\hline Variável & Descrição & Unidade & Tipo & Fonte \\
\hline Recurso & Recursos totais repassados pelo & Reais (R\$) & Input & CGMAC/DAE/ \\
Recebido & Ministério para a UF & & & SAS/MS \\
Leitos & Leitos cirúrgicos que atendem ao & Número de & Input & CNES/TABNE \\
Cirúrgicos & SUS & leitos & & T \\
Cirurgiões & Médicos cirurgiões gerais que & Número de & Input & CNES/TABNE \\
Gerais & atendem ao SUS & médicos & & T \\
População & População total de referência & Número de & Uncontrolled & CGMAC/DAE/ \\
Alvo & assinalada nos projetos aprovados & habitantes & Input & SAS/MS \\
& & & & TCU/TABNET \\
Cirurgias & Produção hospitalar por & Número de & Output & DATASUS/ \\
Eletivas & procedimentos elencados na Política & procedimentos & & TABWIN \\
Realizadas & & realizados & & \\
\hline
\end{tabular}

Nota. Fonte: Elaborada pelos autores.

Foram utilizadas como variáveis input, ou seja, funcionam como insumos no modelo, constituindo os recursos utilizados no processo: Recurso Recebido - são os recursos totais repassados para UF pelo Ministério da Saúde em Reais; Leitos Cirúrgicos - representa o total de leitos cirúrgicos que atendem ao SUS; e Cirurgiões Gerais - total de médicos cirurgiões gerais que atendem ao SUS. Utilizamos ainda a variável População Alvo, que é a população total de referência constante nos projetos aprovados, sendo essa, por sua vez, uma variável de natureza input não-controlada (uncontrolled input). Já a variável Cirurgias Eletivas Realizadas possui natureza output (resultado). Corresponde, assim, à quantidade de bens e/ou serviços produzidos pela unidade de gestão.

A Tabela 2 apresenta, para 2006, os resultados da análise com utilização do modelo com retornos variáveis de escala e orientação para maximização dos outputs. 
Tabela 2

Análise de Eficiência Relativa: Outputs Observados e Esperados em Função dos Inputs Alocados, Projetos Apresentados e Municípios Envolvidos, por UF, 2006

\begin{tabular}{|c|c|c|c|c|c|c|c|c|c|c|c|}
\hline UF & $\begin{array}{l}\text { Recurso } \\
\text { Recebido }\end{array}$ & $\begin{array}{c}\text { População } \\
\text { Alvo }\end{array}$ & $\begin{array}{l}\text { Cirurgiões } \\
\text { Gerais }\end{array}$ & $\begin{array}{l}\text { Leitos } \\
\text { Cirúrgicos }\end{array}$ & $\begin{array}{l}\text { Cirurgias } \\
\text { Eletivas } \\
\text { Realizadas }\end{array}$ & $\begin{array}{l}\text { Cirurgias } \\
\text { Eletivas } \\
\text { Esperadas }\end{array}$ & Diferença & $\begin{array}{l}\text { Eficiência } \\
\text { Relativa da } \\
\text { Gestão }\end{array}$ & $\begin{array}{l}\text { Referências } \\
\text { Benchmark }\end{array}$ & $\begin{array}{l}\mathrm{N}^{\circ} \text { de } \\
\text { Projetos }\end{array}$ & $\begin{array}{c}\mathrm{N}^{\mathrm{o}} \mathrm{de} \\
\text { Municípios } \\
\text { Envolvidos }\end{array}$ \\
\hline $\mathbf{A L}$ & $6.785 .802,00$ & 3.015 .912 & 413 & 903 & 0 & 13.489 & -13.489 & 0 & 0 & 5 & 102 \\
\hline $\mathbf{A P}$ & $1.337 .820,76$ & 594.587 & 30 & 139 & 0 & 1.526 & -1.526 & 0 & 0 & 1 & 16 \\
\hline $\mathbf{B A}$ & $7.196 .345,50$ & 3.198 .376 & 338 & 1.412 & 12.499 & 14.454 & -1.955 & 86,5 & 0 & 14 & 120 \\
\hline GO & $16.155 .160,00$ & 7.180 .071 & 1.211 & 3.768 & 13.944 & 24.498 & -10.554 & 56,9 & 0 & 12 & 235 \\
\hline MA & $5.452 .535,26$ & 2.423 .349 & 207 & 1.170 & 788 & 8.955 & -8.167 & 8,8 & 0 & 6 & 67 \\
\hline MG & $15.176 .225,00$ & 6.744 .989 & 1.224 & 2.497 & 7.818 & 24.447 & -16.629 & 32 & 0 & 12 & 252 \\
\hline MS & $355.350,00$ & 157.933 & 21 & 80 & 1.148 & 1.148 & 0 & 100 & 14 & 1 & 12 \\
\hline MT & $3.325 .647,76$ & 1.478 .066 & 266 & 654 & 270 & 9.339 & -9.069 & 2,9 & 0 & 3 & 69 \\
\hline $\mathbf{P R}$ & $23.265 .324,76$ & 10.340 .144 & 1.362 & 4.337 & 24.630 & 24.630 & 0 & 100 & 7 & 12 & 399 \\
\hline $\mathbf{R J}$ & $5.282 .335,76$ & 2.347 .705 & 262 & 1.180 & 629 & 11.264 & -10.635 & 5,6 & 0 & 3 & 12 \\
\hline $\mathbf{R N}$ & $7.094 .157,76$ & 3.152 .959 & 399 & 1.417 & 894 & 17.014 & -16.120 & 5,3 & 0 & 10 & 136 \\
\hline
\end{tabular}

Continua 


\section{Talela 2 (continuação)}

\begin{tabular}{|c|c|c|c|c|c|c|c|c|c|c|c|}
\hline UF & $\begin{array}{l}\text { Recurso } \\
\text { Recebido }\end{array}$ & $\begin{array}{c}\text { População } \\
\text { Alvo }\end{array}$ & $\begin{array}{l}\text { Cirurgiões } \\
\text { Gerais }\end{array}$ & $\begin{array}{c}\text { Leitos } \\
\text { Cirúrgicos }\end{array}$ & $\begin{array}{c}\text { Cirurgias } \\
\text { Eletivas } \\
\text { Realizadas }\end{array}$ & $\begin{array}{l}\text { Cirurgias } \\
\text { Eletivas } \\
\text { Esperadas }\end{array}$ & Diferença & $\begin{array}{l}\text { Eficiência } \\
\text { Relativa da } \\
\text { Gestão }\end{array}$ & $\begin{array}{l}\text { Referências } \\
\text { Benchmark }\end{array}$ & $\begin{array}{c}\mathrm{N}^{\mathrm{o}} \mathrm{de} \\
\text { Projetos }\end{array}$ & $\begin{array}{l}\quad \mathrm{N}^{\mathrm{o}} \text { de } \\
\text { Municípios } \\
\text { Envolvidos }\end{array}$ \\
\hline $\mathbf{R R}$ & $489.146,26$ & 217.398 & 11 & 71 & 273 & 273 & 0 & 100 & 1 & 1 & 15 \\
\hline $\mathbf{R S}$ & $8.772 .537,50$ & 3.898 .906 & 574 & 1.628 & 24.360 & 24.360 & 0 & 100 & 19 & 1 & 421 \\
\hline SC & $13.199 .778,00$ & 5.866 .568 & 702 & 2.371 & 10.954 & 24.404 & -13.450 & 44,9 & 0 & 1 & 293 \\
\hline SE & $4.427 .529,76$ & 1.967 .791 & 308 & 911 & 73 & 12.378 & -12.305 & 0,6 & 0 & 4 & 75 \\
\hline SP & $68.999 .541,80$ & 30.666 .463 & 4.203 & 10.588 & 8.860 & 24.630 & -15.770 & 36 & 0 & 31 & 637 \\
\hline TO & $2.937 .888,00$ & 1.305 .728 & 122 & 530 & 0 & 5.387 & -5.387 & 0 & 0 & 1 & 139 \\
\hline Total & 242.035.368,08 & 107.571.275 & 14.278 & 43.994 & 126.689 & 333.089 & -206.400 & 35,3 & & 190 & 3.492 \\
\hline
\end{tabular}

Nota. Fonte: Elaborada pelos autores com os resultados do Frontier Analyst. (2008). (Versão 4) [Software]. Kendal, Cumbria, UK: Banxia Software Ltd., utilizando dados do (Ministério da Saúde - Cadastro Nacional de Estabelecimentos de Saúde. (2006). Recursos Humanos - Profissionais segundo CBO de 2002. Recuperado em 18 de junho de 2010, de http://tabnet.datasus.gov.br/cgi/deftohtm.exe?cnes/cnv/prid02br.def), e relatórios do Ministério da Saúde. (2006). Relatórios DATASUS. CGMAC/DAE/SAS/MS. Recuperado em 14 junho, 2010, de http://msbbs.datasus.gov.br/public/default.htm. Os estados do AC, ES, PA, RO não enviaram projetos no ano de 2006, por isso não constam na tabela. 
Das 23 UFs avaliadas em 2006, 04 (17\%) alcançaram uma eficiência relativa ótima (100\%), no sentido de Pareto, sendo elas, em ordem alfabética: Mato Grosso do Sul, Paraná, Roraima e Rio Grande do Sul. Destaque para Rio Grande do Sul e Mato Grosso do Sul, que obtiveram, respectivamente, 19 e 14 referências na curva de eficiência, o que significa, de acordo com a metodologia utilizada, uma UF onde, possivelmente, boas práticas de gestão estão sendo empregadas, tornando-se, portanto, benchmark para as demais.

Alagoas, Amapá e Tocantins (13\% das UFs) tiveram eficiência relativa igual a zero (0\%). Ou seja, apesar de apresentarem projetos e receberem recursos do Ministério da Saúde, não realizaram nenhum procedimento cirúrgico eletivo. Esses estados receberam juntos cerca de R\$ 11 milhões (conforme Tabela 2) e, considerando as demais variáveis inputs, esperava-se que fossem produzidas nessas unidades da federação mais de 20.000 cirurgias eletivas, de acordo com o modelo.

Devemos ressaltar ainda que 8 UFs (34\%) apresentaram eficiência relativa muito baixa, entre $0,1 \%$ e $10 \%$. São elas: Maranhão, Mato Grosso, Paraíba, Pernambuco, Piauí, Rio de Janeiro, Rio Grande do Norte e Sergipe. Juntos, esses estados produziram 101.179 procedimentos a menos do que o esperado pelo modelo, e necessitam de um esforço bastante significativo (superior a 90\%) para alcançar a Fronteira de Eficiência calculada. A Figura 2 confronta as informações sobre o número de cirurgias eletivas esperadas e as de fato realizadas por UF em 2006.

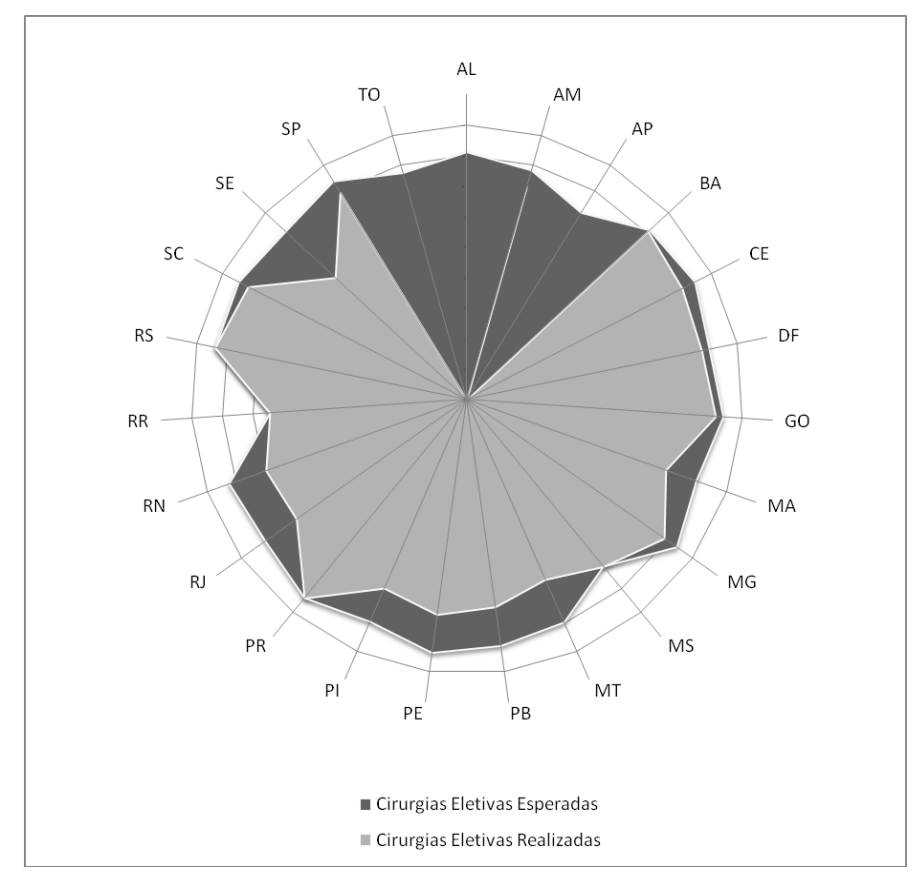

Figura 2. Gráfico com a Distribuição das Cirurgias Eletivas Esperadas versus Cirurgias Eletivas Realizadas por UF, 2006.

Fonte: Elaborada pelos autores com os resultados do Frontier Analyst. (2008). (Versão 4) [Software]. Kendal, Cumbria, UK: Banxia Software Ltd., utilizando dados do CNES (Ministério da Saúde - Cadastro Nacional de Estabelecimentos de Saúde. (2006). Recursos Humanos - Profissionais segundo CBO de 2002. Recuperado em 18 de junho de 2010, de http://tabnet.datasus.gov.br/cgi/deftohtm.exe?cnes/cnv/prid02br.def), e relatórios do Ministério da Saúde. (2006). Relatórios DATASUS. CGMAC/DAE/SAS/MS. Recuperado em 14 junho, 2010, de http://msbbs.datasus.gov.br/public/default.htm.

O diferencial da metodologia DEA é a possibilidade de comparação, não apenas dos valores apurados e ordenados dos índices, mas também de seus valores esperados. Ou seja, o que mais nos interessa no estudo é justamente a diferença entre os valores observados e os valores esperados em destaque na Figura 2. A diferença entre os valores das variáveis baseia-se na hipótese, calculada pelo modelo, de eficiência máxima das DMUs, levando em consideração, de um lado, as combinações possíveis entre inputs e outpus e, de outro, a eficiência relativa e comparada com as práticas das demais DMUs. 
A Figura 3 apresenta o nível de esforço em percentual que as UFs devem realizar para alcançar a Fronteira de Eficiência.

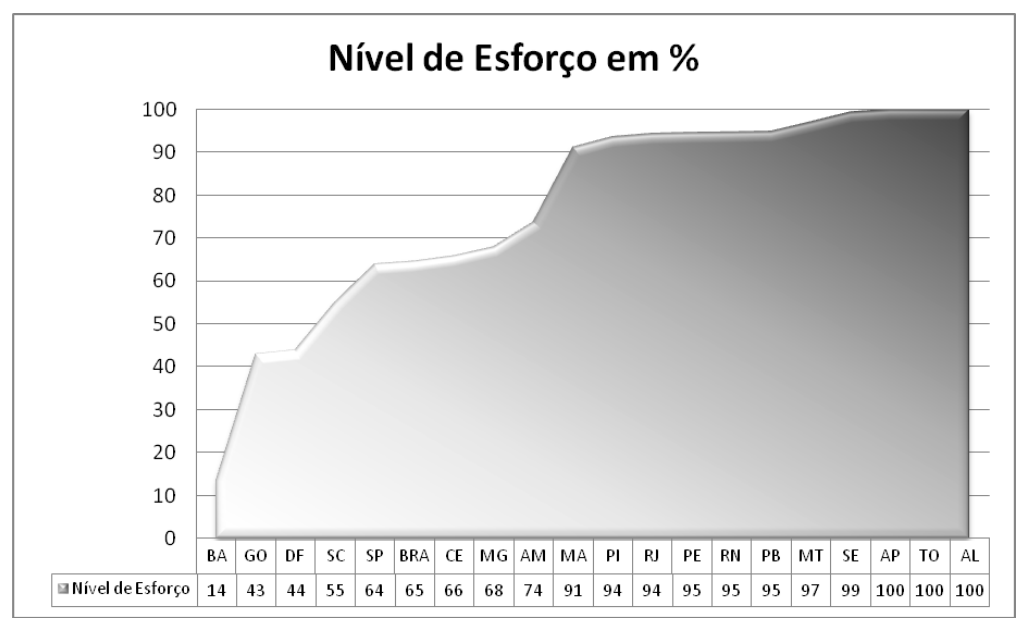

Figura 3. Gráfico com a Distribuição das 'Folgas', em Porcentagem (\%), para Deslocamento em Direção à Fronteira de Eficiência, Nível de Esforço por UF em 2006.

Fonte: Elaborada pelos autores com os resultados do Frontier Analyst. (2008). (Versão 4) [Software]. Kendal, Cumbria, UK: Banxia Software Ltd., utilizando dados do CNES (Ministério da Saúde - Cadastro Nacional de Estabelecimentos de Saúde. (2006). Recursos Humanos - Profissionais segundo CBO de 2002. Recuperado em 18 de junho de 2010, de http://tabnet.datasus.gov.br/cgi/deftohtm.exe?cnes/cnv/prid02br.def), e relatórios do Ministério da Saúde. (2006). Relatórios DATASUS. CGMAC/DAE/SAS/MS. Recuperado em 14 junho, 2010, de http://msbbs.datasus.gov.br/public/default.htm. Os estados do AC, ES, PA, RO não enviaram projetos no ano de 2006, por isso não constam na tabela.

Os resultados apresentados na Figura 3 apontam que 69\% das DMUs avaliadas podem ser consideradas muito ineficientes, pois precisam realizar esforços superiores a 50\% para alcançar a eficiência relativa. E, ao considerarmos as demais UFs, que não configuraram benchmarks, notamos que apenas a Bahia necessita realizar esforço inferior a $20 \%$ para alcançar a fronteira e figurar entre as melhores performances na gestão da Política.

\section{Conclusões}

Apesar do desempenho bastante precário da maioria das UFs na gestão do programa, foi possível identificar alguns avanços recentes em sua formulação, tais como: (a) revisão do elenco de procedimentos cirúrgicos, contemplando, assim, 11 (onze) especialidades (traumato-ortopedia, otorrinolaringologia, oftalmologia, urologia, ginecologia, cirurgia vascular, proctologia, cirurgia geral, mastologia, endocrinologia e gastroenterologia); (b) criação de programas estratégicos, visando ampliar o acesso a determinados procedimentos cirúrgicos eletivos; (c) realização de Oficinas Regionais com ampla participação dos técnicos das secretarias estaduais e municipais de Saúde, com a finalidade de divulgar, orientar e capacitar gestores e outros profissionais para a construção; e (d) desenvolvimento dos projetos locais relativos aos procedimentos cirúrgicos eletivos de média complexidade.

A utilização da metodologia ilustra as potencialidades da DEA para avaliação de programas governamentais e políticas públicas, contratualização de metas, priorização de ações corretivas, etc., na medida em que identifica, por variável, as folgas ou níveis de esforços necessários para o alcance de patamares mais eficientes de gestão.

É importante notar as possibilidades de exploração da metodologia DEA como mecanismo relativamente simples e de fácil implementação, que permite a avaliação comparativa de eficiência. Ademais, a técnica possui características positivas de neutralidade, incentiva a construção e o 
aprimoramento de inventários de recursos e também acolhe participações possíveis e desejáveis de gestores e de agentes na avaliação. Essa participação é desejável dada a estrutura complexa de governança adotada pelo SUS. Ademais, ela permite a qualificação e a determinação de caminhos possíveis para a fronteira de eficiência e para fins de monitoramento de objetivos.

\section{Artigo recebido em 09.05.2011. Aprovado em 25.01.2012.}

\section{Notas}

${ }^{1}$ Versão preliminar apresentada no IV Encontro de Administração Pública e Governança da ANPAD, que ocorreu em Vitória/ES nos dias 28 a 30 de novembro de 2010.

${ }^{2}$ Para ver em detalhes a evolução e o funcionamento dos SUS, recomendamos Paim, J., Travassos, C., Almeida, C., Bahia, L., \& Macinko, J. (2011). O sistema de saúde brasileiro: história, avanços e desafios. Saúde no Brasil. The Lancet. Série Especial, 377(9779), 1778-1797. doi: 10.1016/S0140-6736(11)60054-8. O sistema de saúde brasileiro: história, avanços e desafios.

${ }^{3}$ Os estados devem apresentar Projetos que contemplem alguns aspectos preconizados no Pacto de Gestão. São eles: a regionalização e o fortalecimento da rede de serviços de saúde. Esses aspectos devem orientar a descentralização das ações e serviços de saúde, e os processos de negociação e pactuação entre os gestores. Os principais instrumentos de planejamento da Regionalização são o Plano Diretor de Regionalização (PDR), o Plano Diretor de Investimento (PDI) e a Programação Pactuada e Integrada da Atenção à Saúde (PPI) (Portaria $n^{\circ}$ 399, 2006). No SUS, as redes conformam a regionalização da atenção, respeitando a hierarquização de serviços e visando dar conta da atenção integral. Os procedimentos assistenciais utilizados nessas redes são categorizados quanto ao seu grau de incorporação tecnológica em procedimentos de baixa, média e alta complexidade. Tal categorização é adotada de forma correlata pelo Ministério da Saúde para denominar os chamados níveis de atenção à saúde. Pode-se dizer que o objetivo das redes seja garantir a integralidade da atenção e facilitar o acesso aos diversos itinerários diagnósticos e terapêuticos, nelas estruturados de acordo com as condições nosológicas e grupos sociais prioritários.

${ }^{4}$ Recursos de custeio da esfera federal, destinados às ações e serviços de saúde, que configuram o Teto Financeiro Global (TFG), cujo valor, para cada estado e cada município, é definido com base na Programação Pactuada Integrada (PPI). O teto financeiro do estado contém os tetos de todos os municípios, habilitados ou não a qualquer uma das condições de gestão.

${ }^{5}$ A Comissão Intergestores Bipartite (CIB) é uma instância colegiada de decisão do Sistema Único de Saúde (SUS) estadual, integrada paritariamente pelas Secretarias Estaduais de Saúde e por representantes dos secretários municipais de Saúde, por estado.

${ }^{6}$ Para o cálculo da eficiência relativa das UFs na gestão da Política Nacional de Procedimentos Cirúrgicos Eletivos de Média Complexidade foi utilizado o software Frontier Analyst ${ }^{\circledR}$ (2008) versão 4. O programa calcula a eficiência e redefine a medida de desempenho das DMUs com uma análise da fronteira (valores observados e esperados). O programa oferece a opção de calcular a eficiência nos modelos de retornos constantes de escala ou retornos variáveis de escala, nos dois casos com orientação para inputs ou outputs.

\section{Referências}

Afonso, A., \& Aubyn, M. St. (2005). Non-parametric approaches to education and health expenditure efficiency in OECD Countries. Journal of Applied Economics, 8(2), 227-246.

Almeida, V. P. (2006). Avaliação de programas sociais: de mensuração de resultado para uma abordagem construtivista. Pesquisas e Práticas Psicossociais, 1(2), 1-13.

Banker, R., Charnes, A., \& Cooper, W. (1984). Some models for estimating technical and scale inefficiencies in data envelopment analysis. Management Science, 30(9), 1078-1092. doi: 10.1287/mnsc.30.9.1078

Calvo, M. C. M. (2005). Análise da eficiência produtiva de hospitais públicos e privados no Sistema Único de Saúde (SUS). In S. F. Piola \& E. L. Jorge (Orgs.), Economia da saúde: $1^{\circ}$ Prêmio Nacional-2004: coletânea premiada (pp. 133-161). Brasília: IPEA, DFID. 
Castro, C. E. T. (2003). Avaliação da eficiência gerencial de empresas de água e esgotos brasileiras por meio da envoltória de dados (DEA) (Dissertação de mestrado). Pontifícia Universidade Católica do Rio de Janeiro, Rio de Janeiro, RJ, Brasil.

Charnes, A., Cooper, W., \& Rohdes, E. (1981). Evaluating program and managerial efficiency: an application of data envelopment analysis to program follow through. Management Science, 27(6), 688-697. doi: 10.1287/mnsc.27.6.668

Chilingerian, J. A. (1994). Exploring why some physicians hospital practices are more efficient: taking DEA inside the hospital. In A. Charnes, W. W. Cooper, A. Y. Lewin, \& L. M. Seiford (Eds.), Data envelopment analysis (pp. 167-194). London: Kluwer Academic Publishers.

Debreu, G. (1951). The coefficient of resource utilization. Econometrica, 19(3), 273-292. doi: $10.2307 / 1906814$

Faria, C. A. P. (2005). A política da avaliação de políticas públicas. Revista Brasileira de Ciências Sociais, 20(59), 97-109. doi: 10.1590/S0102-69092005000300007

Faria, F. P., Jannuzzi, P. M., \& Silva, S. J. (2008). Eficiência dos gastos municipais em saúde e educação: uma investigação através da análise envoltória no estado do Rio de Janeiro. Revista de Administração Pública, 42(1), 155-177. doi: 10.1590/S0034-76122008000100008

Farrell, M. J. (1957). The measurement of productive efficiency. Royal Statistical Society, 120(3), 253-281. doi: 10.1016/S0377-2217(01)00022-4

Ferreira, M. P., \& Pitta, M. T. (2008). Avaliação da eficiência técnica na utilização dos recursos do sistema único de saúde na produção ambulatorial. São Paulo em Perspectiva, 22(2), 55-71.

Figueiró, A. C., Frias, P. G., \& Navarro, L. M. (2010). Avaliação em saúde: conceitos básicos para a prática nas instituições. In I. Samico, E. Felisberto, A. C. Figueiró, \& P. G. Frias (Orgs.), Avaliação em saúde: bases conceituais e operacionais (pp. 1-13). Rio de Janeiro: MedBook.

Frontier Analyst. (2008). (Versão 4) [Software]. Kendal, Cumbria, UK: Banxia Software Ltd.

Guba, E. G., \& Lincoln, Y. S. (1989). The coming of age of evaluation. In E. G. Guba \& Y. S. Lincoln. Fourth generation evaluation (pp. 21-49). Newbury Park, CA: SAGE Publications.

Hadad, S., Hadad, Y., \& Simon-Tuval, S. (in press). Determinants of healthcare system's efficiency in OECD countries. The European Journal of Health Economics. doi: 10.1007/s10198-011-0366-3

Joumard, I., André, C., \& Nicq, C. (2010). Health care systems: efficiency and institutions [Working Paper $\mathrm{N}^{\mathrm{o}}$ 769]. OECD Economics Department, Paris, França.

La Forgia, G. M., \& Couttolenc, B. F. (2008) Hospital performance in Brazil: the search for excellence. Washington, DC: The World Bank.

Lei n. 8.080, de 19 de setembro de 1990. (1990). Dispõe sobre as condições para a promoção, proteção e recuperação da saúde, a organização e o funcionamento dos serviços correspondentes e dá outras providências. Diário Oficial da União. Brasília, DF: Congresso Nacional.

Lins, M. E., \& Meza, L. A. (2000). Análise envoltória de dados e perspectivas de integração no ambiente do apoio à decisão. Rio de Janeiro: Editora Coppe/UFRJ.

Lins, M. E., Lobo, M. S. C., Silva, A. C. M., Fiszman, R., \& Ribeiro, V. J. P. (2007). O uso da análise envoltória de dados (DEA) para avaliação de hospitais universitários brasileiros. Ciência \& Saúde Coletiva, 12(4), 985-998. doi: 10.1590/S1413-81232007000400020 
Machado, F., \& Simões, A. (2008). Análise custo-efetividade e índice de qualidade da refeição aplicados à estratégia global da OMS. Revista de Saúde Pública, 42(1), 64-72. doi: 10.1590/S0034-89102008000100009

Marinho, A. (2000). Hospitais universitários: avaliação comparativa de eficiência técnica. Economia Aplicada, 4(2), 315-349.

Marinho, A. (2001). Estudo da eficiência em alguns hospitais públicos e privados com a geração de rankings [Texto para discussão, $\mathrm{N}^{\circ}$ 794]. Brasília: IPEA.

Marinho, A. (2003). Avaliação da eficiência técnica nos serviços de saúde nos municípios do Rio de Janeiro. Revista Brasileira de Economia, 57(3), 515-534. doi: 10.1590/S003471402003000300002

Marinho, A., Cardoso, S. S., \& Almeida, V. V. (2011). Brasil, América Latina e Caribe: avaliação de eficiência em sistemas de saúde [Texto para discussão, $\mathrm{N}^{\circ}$ 1646]. Brasília: IPEA.

Marinho, A., \& Façanha, L. (2002). Hospitais universitários: indicadores de utilização e análise de eficiência técnica. Economia Aplicada, 6(3), 607-638.

Ministério da Saúde. (2006). Relatórios DATASUS. CGMAC/DAE/SAS/MS. Recuperado em 14 junho, 2010, de http://msbbs.datasus.gov.br/public/default.htm.

Ministério da Saúde. (2008). Relatório de gestão atenção especializada média e alta complexidade 2000 - 2005 (Série C. Programas, Projetos e Relatórios). Brasília: Autor.

Ministério da Saúde. (2009). O SUS de A a Z: garantindo saúde nos municípios (3a ed.). Brasília: Autor.

Ministério da Saúde - Cadastro Nacional de Estabelecimentos de Saúde. (2006). Recursos Humanos Profissionais segundo $C B O$ de 2002. Recuperado em 18 junho, 2010, de http://tabnet.datasus.gov.br/cgi/deftohtm.exe?cnes/cnv/prid02br.def

Parkin, D., \& Hollingsworth, B. (1997). Measuring production efficiency of acute hospitals in Scotland, 1991-1994: validity issues in data envelopment analysis. Applied Economics, 29(11), 1425-1433. doi: 10.1080/000368497326255

Portaria n. 252 GM, de 06 de fevereiro de 2006. (2006). Redefine a política nacional de procedimentos cirúrgicos eletivos de média complexidade. Diário Oficial da União. Brasília, DF: Ministério da Saúde.

Portaria n. 399 GM, de 22 de fevereiro de 2006. (2006). Divulga o pacto pela saúde 2006 consolidação do SUS e aprova as diretrizes operacionais do referido pacto. Diário Oficial da União. Brasília, DF: Ministério da Saúde.

Proite, A., \& Souza, M. C. S. (2004, dezembro). Eficiência técnica, economias de escala, estrutura da propriedade e tipo de gestão no sistema hospitalar brasileiro. Anais Encontro Nacional de Economia da Associação Nacional de Centros de Pós-Graduação em Economia, João Pessoa, $\mathrm{PB}$, Brasil, 32.

Reis, C. O., Ribeiro, J. A., \& Piola, S. (2001). Financiamento das políticas sociais nos anos 1990: o caso do Ministério da Saúde [Texto para discussão, No 802]. Brasília: IPEA.

Rouse, P., Harrison, J., \& Chen, L. (2010). Data envelopment analysis: a practical tool to measure performance. Australian Accounting Review, 20(2), 165-177. doi: 10.1111/j.18352561.2010.00090.x 
Shafritz, J. M., Russell, E. W., \& Borick, C. P. (2007). Introducing public administration. New York: Pearson Education.

Smith, P. C., \& Street, A. (2005). Measuring the efficiency of public services: the limits of analysis. Journal of the Royal Statistical Society: Series A (Statistics in Society), 168(2), 401-417. doi: 10.1111/j.1467-985X.2005.00355.x 